\title{
Pathogen population structure can explain hospital outbreaks
}

\author{
Fabrizio Spagnolo $\mathbb{1}^{1} \cdot$ Pierre Cristofari ${ }^{2,3} \cdot$ Nicholas P. Tatonetti $\mathbb{1}^{2,4,5} \cdot$ Lev R. Ginzburg ${ }^{6}$ - Daniel E. Dykhuizen ${ }^{7}$
}

Received: 30 May 2018 / Accepted: 22 June 2018 / Published online: 25 July 2018

(c) International Society for Microbial Ecology 2018

\begin{abstract}
Hospitalized patients are at risk for increased length of stay, illness, or death due to hospital acquired infections. The majority of hospital transmission models describe dynamics on the level of the host rather than on the level of the pathogens themselves. Accordingly, epidemiologists often cannot complete transmission chains without direct evidence of either host-host contact or a large reservoir population. Here, we propose an ecology-based model to explain the transmission of pathogens in hospitals. The model is based upon metapopulation biology, which describes a group of interacting localized populations and island biogeography, which provides a basis for how pathogens may be moving between locales. Computational simulation trials are used to assess the applicability of the model. Results indicate that pathogens survive for extended periods without the need for large reservoirs by living in localized ephemeral populations while continuously transmitting pathogens to new seed populations. Computational simulations show small populations spending significant portions of time at sizes too small to be detected by most surveillance protocols and that the number and type of these ephemeral populations enable the overall pathogen population to be sustained. By modeling hospital pathogens as a metapopulation, many observations characteristic of hospital acquired infection outbreaks for which there has previously been no sufficient biological explanation, including how and why empirically successful interventions work, can now be accounted for using population dynamic hypotheses. Epidemiological links between temporally isolated outbreaks are explained via pathogen population dynamics and potential outbreak intervention targets are identified.
\end{abstract}

\section{Introduction}

Electronic supplementary material The online version of this article (https://doi.org/10.1038/s41396-018-0235-5) contains supplementary material, which is available to authorized users.

\section{Fabrizio Spagnolo}

fs2599@columbia.edu

1 Ecology, Evolution and Environmental Biology Department, Columbia University, New York, NY 10027, USA

2 Department of Biomedical Informatics, Columbia University Medical Center, New York, NY 10032, USA

3 Astronomy Department, Columbia University, New York, NY 10027, USA

4 Department of Systems Biology, Columbia University Medical Center, New York, NY 10032, USA

5 Department of Medicine, Columbia University, New York, NY 10032, USA

6 Applied Biomathematics, Setauket, NY 11733, USA

7 Department of Ecology and Evolution, Stony Brook University, Stony Brook, NY 11794, USA

Hospital acquired infections (HAIs) adversely impact patient care and outcomes. Worldwide, the rate of HAIs for patients in the intensive care unit approaches 30\% and deaths due to antibiotic resistant pathogens are expected to outpace even cancer deaths and reach 10 million annually by 2050 [1]. Central to the fight to lower the number of HAIs are ways in which we can lower or slow transmission of pathogens within hospital environments [2]. Hospitals have found some success in doing this using a variety of mostly behavioral programs. As a result, patient isolation [3], patient [4], and environmental disinfection [5], as well as handwashing protocols [6], remain the most successful and cost effective means of controlling transmission of HAIs.

In recent years, the application of genomic tools to hospital epidemiology has also become a valued outbreak control tool, enhancing both magnification and resolution into outbreaks [7]. However, even as genomic analyses coupled with epidemiological data have provided a deeper understanding of how nosocomial infections spread in hospital outbreaks, there remains two interacting aspects of 


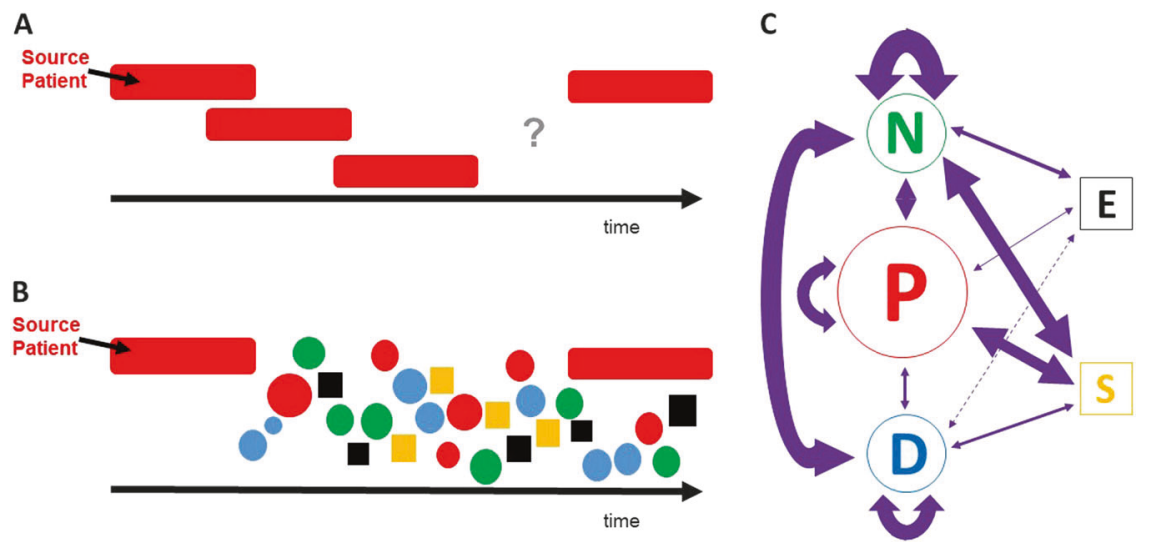

Fig. 1 The Ephemeral Island Metapopulation Model in Hospital Environments. Colors represent each patch class: Patients in red; Nurses in green; Doctors in blue; Equipment in black; and Stationary surfaces are yellow. a A Gantt-like diagram of a hospital outbreak with overlap of identified infected patients (elongated red bars) over time. The undefined area denoted by the question mark is a discontinuity in the epidemiological analysis of transmission typical of outbreaks. b A similar depiction of a hospital outbreak under the proposed model,

outbreaks that epidemiologists still do not understand well: the discontinuity in outbreak cases and the lack of known pathogen reservoirs. The discontinuity of infections in outbreaks are periods of time in which there is a gap between recognized cases. There is an outbreak, then new cases cease, in some cases for extended periods of time, leading epidemiologists thinking the outbreak has subsided. But often, additional infections are later identified [7-11], without any epidemiological link between the infected hosts (such as asymptomatic carriers). Genomics has helped because this discontinuity can sometimes be at least partially explained by genomic data and surveillance monitoring during and after outbreaks, such as in the identification of previously unknown infections [8].

When new cases are identified after such breaks and the pathogen is matched to a previous outbreak strain, a reservoir population is presumed to exist [2]. The location and size of the reservoir population is often never identified. Even so, some assumptions about reservoirs are common. For instance, reservoirs are thought to be large populations [12-14], although there are cases in which populations of unknown but lesser size are called reservoirs [2, 15]. In each of these situations, however, the source population is considered to be one from which many potential infections can be founded. This presupposes that the reservoir is a source population, with individual infections being sink populations. Assumptions concerning such source-sinks may turn out to be incorrect.

Another assumption concerning reservoirs is that they are durable, self-supporting, and exist for extended periods of time [16, 17]. Long term sources have been associated with infections such as Legionnaires Disease [18], which is where many ephemeral islands can bridge the discontinuity so long as the metapopulation exists within the hospital facility. c Graphic representation of transmission routes in the model. Transmission route arrows and patch areas represent (approximately) the strength of transmissions modeled. Transmission routes are in linear scale and patch classifications are in log scale (relative to each other). Dashed transmission line between Doctors and Equipment denotes low interaction

often associated with bacterial contamination in water supply systems. Recent studies have begun to elucidate the role of the hospital environment on the existence and persistence of possible nosocomial reservoir populations [1924]. However, these environmental hospital populations violate the assumption that reservoirs are large. Further, the duration of these small populations, while longer than previously suspected, is by no means permanent [25].

This necessitates the possibility that hospital acquired infections can be caused by pathogens from ephemeral environmental populations [26], even when these populations are small. Importantly, if a pathogen from one of these populations is capable of causing an infection in a susceptible host, then that pathogen is also capable of founding a new environmental population somewhere else within the hospital environment, with the size, growth rate, and duration of that population being dependent upon a series of independent factors, not the least of which is how well adapted the bacterial clone is to surviving under such conditions and how often that population's location is cleaned by facility staff.

The resulting conditions are such that hospital pathogens can survive for prolonged periods of time by emigrating from one ephemeral population to another until one of three possible outcomes is reached: bacterial cells will eventually die off, be killed through the regular process of hospital cleaning, or cause a new infection. If the cells are able to infect a new host, the size of the infecting pathogen population will grow exponentially and many new populations can be founded, starting the process a new. The result is that the pathogen population survives as a metapopulation (Fig. 1a, b). 
Here, we propose a model for HAIs that is based upon the concept of a metapopulation [27] and is further informed by island biogeography [28, 29]. This differs from most outbreak models by departing from the SusceptibleInfected-Recovered (SIR) focus on hosts and moving our attention to the population biology of the pathogen itself. We should note, however, that hosts can be viewed as patch populations in SIR models [30]. For our purposes here, we will presume the pathogen to be bacterial. We propose that hospital pathogen metapopulations survive across a patchy distribution of habitable areas within a larger inhabitable landscape by existing in smaller populations for some period of time. Individuals from one patch (or island, the terms will be interchangeable for our purposes) move to other locales after some period of time before either the resources required to survive in the original patch are exhausted or that patch is wiped out by cleaning and the resident population goes extinct. In this way, populations exist within a particular hospitable patch for a period of time, but not indefinitely. The patches can vary spatially, like small islands in an inhospitable sea, or temporally, such as a blooming plant in a desert might for a group of insect nomads. Then, after some unknown amount of time has passed, individuals from an island population either die off or move on to other islands, within which they can found a new island population or join one already existing. Additionally, in our model, migration distances are replaced with a sort of network connectivity measure dependent upon the amount and intensity of interactions between island types. In macroscopic species, migration probability is a function of physical distance, whereas for microbes, the ability to go from patch to patch is really a matter of how you will be carried there or by whom.

The applicability of metapopulations to problems of pest control [31], parasitism, and infections [32] has been recognized for some time. The further applicability of metapopulation ecology to nosocomial infection transmission should also be evident. However, in the hospital environment instead of naturally occuring limits on patch viability, the likelihood of an island being sustainable or not is dictated by human behaviors such as hand washing, patient isolation, antibiotic stewardship protocols, healthcare worker cohorting, and the frequency of environmental cleaning [33]. Not surprisingly, all of these behaviors are also intervention targets thought to be effective infection control measures [24, 33-35]. Here, we provide an adequate scientific base capable of explaining why [36, 37]. Understanding metapopulation dynamics of pathogens in this way has the potential to provide a wide scientific foundation upon which hospital epidemiology can be interpreted and further developed.

\section{Methods}

Our model investigates the ability of pathogens to spread and survive via numerous interactions between different carriers. Using Monte Carlo simulation, we model the interactions between different islands and investigate the long term viability of the pathogen metapopulation. The model considers five classes of island/patch. Three of these are potential human hosts: patients $(\mathrm{P})$, nurses $(\mathrm{N})$, and physicians (D). Two account for the environment: mobile equipment (E) and non-mobile surfaces (S), modeling the role of the environment in the potential survival and transmission of HAI pathogens. Through these five classes most typical interactions can be simulated. The island classes are defined by the behavior and levels of interaction relative to each class type within the model system. This means that not all members of a class necessarily perform the same job function within the hospital, only that they behave in a manner similar to the defining characteristic of their class [38]. Therefore, not all members of the Nurse island class are nurses; they may be a Physical Therapist or an X-ray Technician, but their level and number of interactions are similar enough to place them in the same class as a Nurse. In the present study, efforts were undertaken to keep the model as simple as possible. For this reason, the number of classes is limited here. However, the design of the model allows for the modular addition of more classes by expanding the relevant matrices and incorporating into them a greater number of parameter values (see below).

General properties of interactions for each island type can be listed as follows: (D) interact with all patients (P). $(\mathrm{N})$ do not necessary interact with all $(\mathrm{P})$, but the frequency of $(\mathrm{N})-(\mathrm{P})$ interactions is greater than the frequency of $(\mathrm{P})$ (D). (S) do not directly interact with each other. (P) only have direct interaction with (E) and (S) in their immediate environment. (S) and (E) can be cleaned more effectively than $(\mathrm{N}),(\mathrm{P})$, and (D) (Fig. 1c).

Several parameters are needed to simulate the pathogen population over the different islands. The parameter $\operatorname{Max}_{i}$ is a vector that corresponds to the carrying capacity an island of type $i$ can support. The number of interactions, over a given period of time, between an island of type $i$ and an island of type $j$ is noted in matrix form as $N_{i j}$. The probability of pathogen transmission, i.e., the probability for the pathogen to be found on $j$ after an interaction with $i$ constitutes the matrix, $P_{i j}$. Additional parameter classifications can be added by incorporating new columns into the matrices, thereby affording the model a level of customizability. The frequency of cleaning an island per unit time is a vector, $C_{i}$. The efficacy of cleaning, i.e., the fraction of the patch population killed through a cleaning event is the vector $L_{i}$. Values for these parameters as used here are given 


$$
\begin{aligned}
& N_{i j}\left(\begin{array}{ccccc}
10 & 16 & 3.75 & 0 & 5.64 \\
& 48 & 11.86 & 6 & 20.06 \\
& & 4 & 1 & 16 \\
& & & 2 & 0 \\
& & & & 0
\end{array}\right) \\
& P_{i j}\left(\begin{array}{ccccc}
0.04 & 0.08 & 0.17 & 0 & 0.106 \\
& 0.15 & 0.17 & 0.05 & 0.106 \\
& & 0.05 & 0.1 & 0.106 \\
& & & 0.11 & 0 \\
& & & & 0
\end{array}\right) \\
& \operatorname{Max}_{i}=\left(\begin{array}{lllll}
10^{6} & 10^{6} & 10^{10} & 10^{4} & 10^{4}
\end{array}\right) \\
& C_{i}=\left(\begin{array}{lllll}
2 & 6 & 2 & 1 & 1
\end{array}\right) \\
& L_{i}=\left(\begin{array}{lllll}
0.6 & 0.6 & 0.6 & 0.95 & 0.95
\end{array}\right)
\end{aligned}
$$

Fig. 2 Parameter values used for the current simulations. The matrices $N_{i j}$ and $P_{i j}$ represent the number of interactions within and between parameter classes and the level of transfer of live bacterial cells during the interaction, respectively. The $N_{i j}$ matrix contains number of interactions per $8 \mathrm{~h}$ shift and is based upon publicly available data sources for a range of health care facilities. The carrying capacity for any class of island is dependent upon patch type but is impacted by the frequency of cleaning $\left(C_{i}\right)$, as well as the thoroughness of that cleaning $\left(L_{i}\right)$. The regularity and thoroughness of cleaning is expected to keep the metapopulation from reaching equilibrium on a consistent basis, raising the overall importance of the level of detectability of a pathogen within a facility in the ability of that pathogen to persist over time. The parameter classes in the matrices, in order, are: doctor, nurse, patient, equipment, surfaces. Supporting references for parameter values can be found in the SI

in Fig. 2 and are calculated based upon a review of the literature from studies worldwide (see SI for more information). This was done in order to highlight the universal applicability of the model; however, we note that parameter values for a specific clinical site would be necessary for a more refined study.

The population, when left alone on an island, has a doubling time $\tau$. Here, the value of $\tau$ is $8 \mathrm{~h}$. This generation time was chosen based upon empirical evidence of doubling time for bacteria under environmental conditions and can also be adjusted as necessary. The value of $\tau$ here has the added benefit of matching with what we describe as a hospital shift, i.e., the amount of time a particular group of health care workers surround a patient. Thus, simplifying the model in the current instance as the number of interactions between island types are all per shift. See SI for additional discussion of the $\tau$ value used here.

At the beginning of our simulations, all islands are free of pathogens. We introduce a pathogen on a patient for a given amount of time, 30 days $\left(t_{\text {start }}\right)$, after which this index patient is removed. The value of $t_{\text {start }}$ can be adjusted within the simulations, here 30 days was chosen so as to allow the pathogens to infiltrate the environment and allow each trial to begin with similar conditions. However, we consistently observed environmental saturation to occur in our simulation runs in less than the 30 days. We run our simulations by randomly drawing, every day, a sequence of interactions between islands and of cleaning events, and by following the changes in the growth, death (or cleaning), and locations of the populations of pathogens over time. While the model allows for bacterial colonization of patients (P), no secondary infections were allowed as this would effectively reset the model to Day 0 and the question being addressed here concerns metapopulation persistence.

\section{Results}

A total of 1000 Monte Carlo trials were conducted, each for 2000 days (total of 2,000,000 simulated patient-days). The overall number of trials was determined by verifying that the number be sufficient to ensure that our average results do not depend on the total simulations run. We simulate a situation where an index patient carrying a pathogen is admitted to a naïve hospital ward for $t_{\text {start }}=30$ days. Sensitivity analyses indicate that all results are robust to twofold increases/decreases to interaction matrix $\left(N_{i j}\right)$ values (see SI for more information).

All simulations begin with a single index patient. Trials show all patches quickly reaching maximum carrying capacity and remaining at those levels for the duration of the infected patient's stay. Following removal of the source population, island population sizes decline, but do not go extinct for extended periods of time. This results in the continued transmission of the pathogen between hospital workers, environmental patches, and novel patients during that period. Without a new source population (second index patients were not introduced in trials and secondary infections were not allowed), simulations indicate that the metapopulation will eventually go extinct, but the time to total metapopulation extinction in the model is a function of the frequency and level of cleaning events affecting individual patch populations.

This is the first of several unexpected results of our model simulations. The longer a pathogen metapopulation can remain extant within a hospital, the greater the probability of a secondary infection occurring in a susceptible patient. Within our model, any secondary infection would represent a stochastic jackpot event in which a limited number of cells found what will become a large new island population with which other potential hosts (short and long term) are expected to interact. When such a secondary infection occurs, patch populations quickly reach carrying capacity once again, thereby resetting the time to extinction clock for the metapopulation as a whole. As a result, the metapopulation can remain in existence indefinitely. From an epidemiological perspective, there need not be any direct contact at all between the primary and secondary infected 


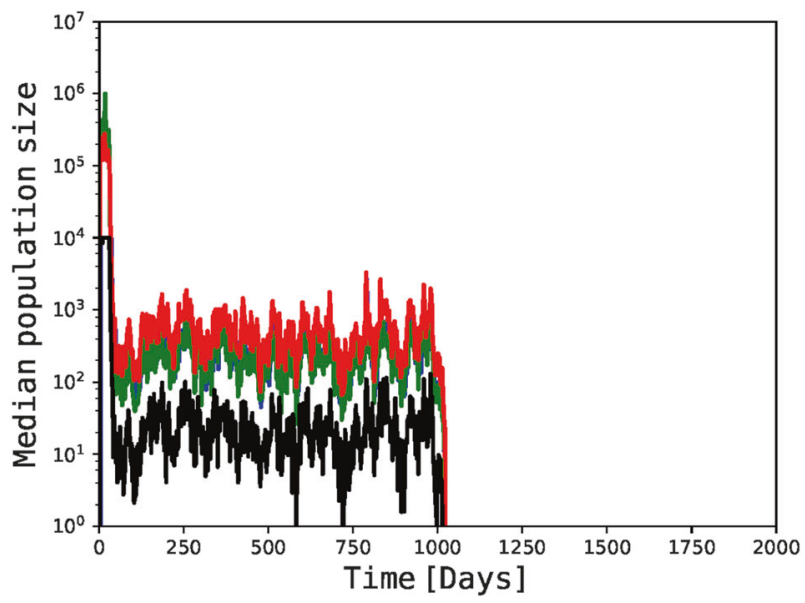

Fig. 3 Median size of patch populations over time for $1000 \mathrm{compu}-$ tational simulation trials. After the initial forcing period, median patch population size fluctuates but remains at a low level for extended periods of time, here over 1000 days. The metapopulation persists at an equilibrium between patch population births and deaths, with the rates of each affected by transmission and cleaning. At any point during this time period, a jackpot even could occur which results in a secondary active infection that would start a new forcing period. Median population size for surface patches (in yellow, not visible) remains close to 0 throughout timeframe. After approximately 1000 days, the other patch type populations also persist at extremely small median sizes. Median time to metapopulation extinction in our simulation trials was 506 days

patients, so long as the time between the two cases is less than the time to metapopulation extinction and there exists a transmission path, regardless of how indirect, between the two infected patients. The reason for the extended persistence of the metapopulation is that in order for permanent extinction of the metapopulation to occur, all islands must be extinct at the same moment (Fig. 3). If any island population remains, regardless of the number of individuals, the possibility remains for other islands to be repopulated over time and for additional infections to occur. In this manner, the model explains the discontinuity of infections often observed in hospital outbreaks.

A second outcome of model simulations relates to the small size of island populations during the period between loss of the source population and extinction. Model parameterization set limits to patch population sizes based upon island class. These limits are in line with empirical data currently available $[6,26,39,40]$. In most simulation trials, the size of patch populations quickly dropped below carrying capacity once the source population was removed (Fig. 4). The island populations remained extant at small sizes for significant periods of time before going extinct. However, the local populations continue to survive during this time, even at such small scales. Patches surviving at levels indicated by our simulations suggest that many of these islands would be too small to be readily detected by

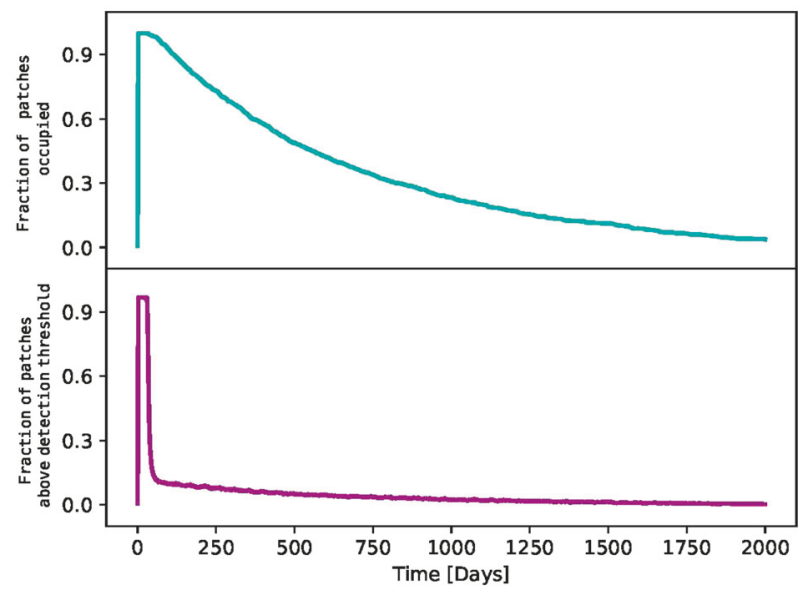

Fig. 4 Fraction of occupied patches over time as compared to fraction of populations above threshold of detectability. A comparison of the average fraction of patches occupied, regardless of local population size, and the fraction of patches with local population sizes greater than the threshold of detectability, here set to $10^{3}$ cells/patch. The slow decline in metapopulation (first panel) suggests that hospital pathogens survive for much longer periods of time than previously suspected without a high probability of detection through most monitoring protocols. For instance, at 500 days, approximately $50 \%$ of all patches have at least some pathogens in them, but only about $15 \%$ have more than the number required to be detected with any reasonable probability

most pathogen sampling techniques currently in use in healthcare facilities.

Typically, hospitals employ culture-based protocols for the presence/absence detection of pathogens. However, all such methods have a lower limit to their resolution of detection [2, 41]. In large part, this limit may be due to sampling itself: in order to detect a pathogen, staff must first swab then culture a strain. Successful detection is impacted by, among other things, the likelihood of sampling enough of the relevant bacteria (typically from a patient). If the same techniques are applied for surveillance of staff and environment during which island populations exist in small populations, the probability of detection is greatly diminished, leading to a large false negative bias. In fact, our simulations suggest that metapopulations can persist on islands for extended periods below a reasonable size threshold of detectability, providing faulty data upon which facility personnel may base critical decisions.

An additional result indicates that a metapopulation model, while robust to changes in interaction or transmission parameters, is sensitive to the number of local populations that make up the metapopulation system. For patch classes such as doctors, nurses, and the patients themselves, the number of possible patches is relatively limited: most (but not all) pathogen islands will be on hands and most of these will have high frequencies of cleaning with high effectiveness. However, the number and duration of non- 


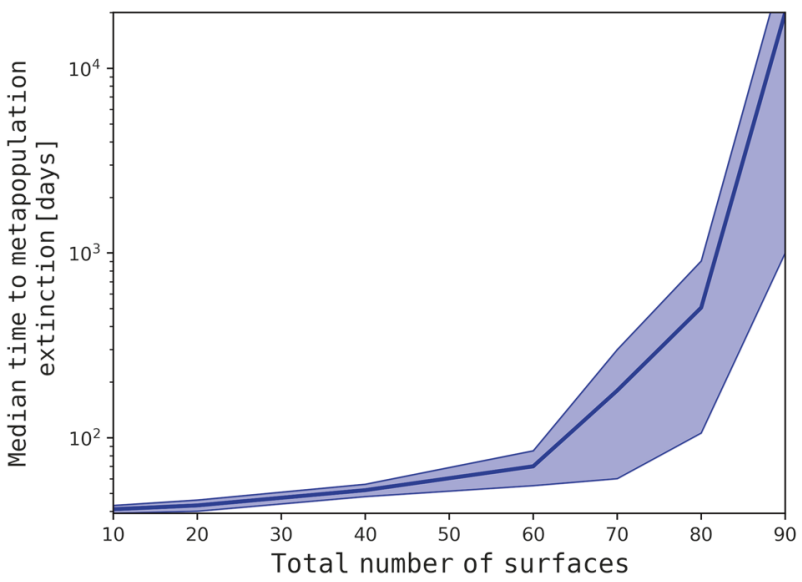

Fig. 5 Importance of the number of surface-type patches. While sensitivity analyses indicate the model to be robust to interaction or transmission values, the total number of patches within a metapopulation is important to the system. Here, the number of possible patches on surfaces must be greater than 60 in order for the metapopulation to resist relatively rapid extinction. The simulations here concern a total of 20 patients and 80 surface patches resulting in only four patches per patient. A larger number of patches per patient can easily be envisioned. Note the Log scale on the $Y$-axis. Shaded area indicates \pm 1 SD

human islands, such as those on equipment, surfaces, and even dust [23] are more problematic. The model here is sensitive to the number of these islands, with minimum thresholds being vital for metapopulation survival (Fig. 5). This may present a unique target for HAI control measures if reliable methods for detecting and monitoring such patches can be implemented in clinical environments. The present simulations all utilized a conservative number of four surface patches per patient. A much larger number of surfaces per patient can be envisioned, including sites known to be easily contaminated such as bedrails, door knobs, toilet handles, call buttons, faucets, etc., suggesting that the median time to extinction for pathogen metapopulations in real-world environments is even longer than that suggested in our simulation trials.

In addition to the sensitivity of the model to patch number, also noteworthy is the model's robustness to the interaction and transmission levels that define the system. By having low sensitivity to changes in these parameters (denoted in $N_{i j}$ and $P_{i j}$ ), simulation trials suggest that a stable equilibrium can be reached for a wide range of physical constraints across hospital conditions. This results in a resilient population-level system by which pathogens can survive and adapt to many different specific healthcare facilities so long as the core structure and function of the hospitals are alike across sites (a similar finding for viral adaptation to novel hosts has been shown previously [42]). The outcome is that hospitals are susceptible to endemic metapopulations of pathogens by their very design and function.

\section{Limitations}

The model presented here incorporates several aspects of established ecological theory, including metapopulation biology and island biogeography theory, and develops them into a framework to potentially explain empirical data concerning nosocomial outbreaks of infectious disease. In the present form, the model should be seen as a baseline, or first-order approximation, of the specific pathogen population dynamics within hospitals. The emphasis here is on attempting to understand the ecology of infectious diseases in the built (and highly structured) environments of healthcare facilities from the perspective of the pathogen as opposed to their hosts while still being cognizant of host biology and interactions.

\section{Model parameter values should be from single site}

Our Sensitivity Analyses (see SI) have shown that the model is robust to changes in most parameter values, however, we highlight in Fig. 5 that the number of surface islands (S) can impact the median time to metapopulation extinction. The number of such surface islands in any hospital, ward, or room, is not clearly known and may be highly specific to any particular facility. As such, we caution that this model, like most models, is limited by the accuracy of the parameter values that inform it.

\section{Model is incomplete}

The model does not take many real-world aspects of HAI into account. For instance, while we mention secondary infections, these are not included in the presented model. In order to do so, the modeler would need to include an additional matrix of the probability of any patient developing a secondary infection. The intent is for this model to be developed by the community over time so as to be properly applicable under correct circumstances.

\section{Discussion}

For almost a century, mathematical models have been applied to epidemic outbreaks [43]. Models are also often used as a mechanism by which we can understand the dynamics of infection transmission and spread in hospital environments [35]. While models have proven helpful in understanding how HAIs spread, they have been limited by our incomplete understanding of the biology that underlies them. For instance, SIR-type models focus on the biology of hosts and make assumptions concerning the pathogen that may limit the applicability to hospital outbreaks. In SIR 
models, the two foundational measures of an outbreak are $\mathrm{R}_{0}$, the average number of secondary infections produced per primary infection, and the infection timescale [30].

Here we propose a model in which pathogen populations in hospital environments act as metapopulations, with small patch populations interconnected by migration between ephemeral islands that interact with each other. The size of these island populations, their duration, and the migration between them are dictated by the human behaviors inherent to the operation of modern healthcare facilities. This provides a biologically relevant basis for observed HAI dynamics and explains empirical data on individual interventions that have correlated with lowered HAI transmissions $[2,5,6,44]$. While many aspects of our model have been observed independently, to our knowledge, they have never been integrated into a common framework such as presented here.

This means that viewing pathogen populations in healthcare environments through a structured population lens provides a system capable of explaining the underlying biology. For instance, evidence of lower HAIs with increased handwashing for health care workers not only makes intuitive sense, but is also supported by the data [6]. In our model, the increased handwashing reflects a change in the frequency of cleaning $\left(C_{i}\right)$ and improved handwashing leads to lower numbers of individual bacteria in the metapopulation $\left(L_{i}\right)$, reducing the overall number of migrating bacteria, having a cascading impact on the number of possible transmission events at all later timepoints for that particular island.

Our simulations indicate that by existing in a metapopulation structure, pathogens can survive within a hospital for extended periods of time even after the original source population is removed. In essence, each patch population contributes to a diffuse reservoir that resists extinction through a bet-hedging strategy in which the metapopulation is never in any one place at any single time. In a SIR-type model framework, this would be equivalent to the infection timescale being greatly extended beyond just the period of measured infections, with the metapopulation capable of founding new infections at any time prior to complete extinction. Surprisingly, the overall metapopulation size remains small meaning that large reservoirs are not required for pathogens to remain extant over long periods. Additionally, the metapopulation reservoir is ultimately a series of sink populations. However, unlike most source-sink systems, the metapopulation may be dependent upon sink populations to found new populations which can then subsequently become sources.

These new source populations would take the form of secondary infections where local patch size can become much larger than that of any other class of patch. In this way, secondary infections represent a kind of stochastic jackpot event in which a small number of individual bacteria found a large new source population capable of resetting the time to metapopulation extinction by saturating the system. This interpretation is rather different from the $\mathrm{R}_{0}$ statistic of SIR models, where one infection will beget a certain number of secondary infections. We acknowledge that our interpretation is an over simplified view that ignores much of infection biology but the underlying tenet that population structure can provide for extended pathogen population existence is supported. Additionally, if the model predictions are correct in real-world scenarios, this would also imply that secondary infections follow not only a single but perhaps several bottleneck events. Consequently, founder effect genetic signatures should be identifiable in hospital outbreaks, as is observed [7, 9], as well as in nosocomial strains more generally, as also seen [ 45 , 46].

\section{Future directions}

The application of an ephemeral island metapopulation model to nosocomial pathogen populations may provide a tractable system for developing and testing intervention hypotheses. The wealth of genomic studies of hospital outbreaks makes population genetic analyses an immediate possibility. Data from such studies can inform experimental exploration of virulence evolution, as well as host-pathogen coevolution. These aspects of the model proposed have the potential to aid the development of the field of evolutionary medicine, partly through the application of evolutionary ecology to the hospital environment. Immediate next steps require informing the model with parameter values specific to individual hospitals to understand the physical limits of metapopulations in specific institutions (individual rooms, wards, entire wings, etc.).

Our model invites study of several aspects of hospital pathogen population biology, with the potential to guide development of novel intervention protocols. This could be accomplished by leveraging the knowledge gained in field biology concerning the conservation of species that live in a metapopulation structure. By reversing the goal from conservation of species to eradication of pathogens, we may be able to positively impact the public health through the manipulation of the very aspects of metapopulation dynamics that prolong persistence (patch size, transmission distance, etc.).

Acknowledgements The authors wish to thank Erik Lavington, Nick Friedenberg, and Emily Rollinson for helpful discussions. FS and PC thank Columbia Frontiers of Science for support, as well as fostering opportunities for interdisciplinary collaboration. This work was funded by Columbia Frontiers of Science Fellowships to FS and PC. 


\section{Compliance with ethical standards}

Conflict of interest The authors declare that they have no conflict of interest.

\section{References}

1. The Review on Antimicrobial Resistance. Tackling drug-resistant infections globally: final report and recommendations. London: United Kingdom; 2016.

2. Palmore TN, Henderson DK. Managing transmission of carbapenem-resistant enterobacteriaceae in healthcare settings: a view from the trenches. Clin Infect Dis. 2013;57:1593-9.

3. Hayden MK, Lin MY, Lolans K, Weiner S, Blom D, Moore NM, et al. Prevention of colonization and infection by klebsiella pneumoniae carbapenemase-producing enterobacteriaceae in long-term acute-care hospitals. Clin Infect Dis. 2015;60:1154-61.

4. Wang X, Panchanathan S, Chowell G. A data-driven mathematical model of CA-MRSA transmission among age groups: evaluating the effect of control interventions. PLoS Comput Biol. 2013;9:e1003328.

5. Boyce JM. Environmental contamination makes an important contribution to hospital infection. J Hosp Infect. 2007;65:50-4.

6. Pittet D, Hugonnet S, Harbarth S, Mourouga P, Sauvan V, Touveneau $\mathrm{S}$, et al. Effectiveness of a hospital-wide programme to improve compliance with hand hygiene. Lancet. 2000;356:1307-12.

7. Snitkin ES, Zelazny AM, Thomas PJ, Stock F, Henderson DK, Palmore TN, et al. Tracking a hospital outbreak of carbapenemresistant Klebsiella pneumoniae with whole-genome sequencing. Sci Transl Med. 2012;4:148ra116.

8. Haller S, Eller C, Hermes J, Kaase M, Steglich M, Radonic A, et al. What caused the outbreak of ESBL-producing Klepbsiella pneumoniae in a neonatal intensive care unit, Germany 2009 to 2012? Reconstructing transmission with epidemiological analysis and whole-genome sequencing. BMJ Open. 2015;5:e07328.

9. Köser CU, Holden MT, Ellington MJ, Cartwright EJ, Brown NM, Ogilvy-Stuart AL, et al. Rapid whole-genome sequencing for investigation of a neonatal MRSA outbreak. N Engl J Med. 2012b;366:2267-75.

10. Chang LWK, Buising KL, Jeremiah CJ, Cronin K, Poy Lorenzo YS, Howden BP, et al. Managing a nosocomial outbreak of carbapenem-resistant Klebsiella pneumoniae: an early Australian hospital experience. Intern Med J. 2015;45:1037-43.

11. Conlan S, Thomas PJ, Deming C, Park M, Lau AF, Dekker JP, et al. Single-molecule sequencing to track plasmid diversity of hospital-associated carbapenemase-producing Enterobacteriaceae. Sci Transl Med. 2014;6:254ra126.

12. Gupta N, Limbago BM, Patel JB, Kallen AJ. Carbapenemresistant Enterobacteriaceae: epidemiology and prevention. Clin Infect Dis. 2011;53:60-7.

13. Lin MY, Lyles-Banks RD, Lolans K, Hines DW, Spear JB, Petrak $\mathrm{R}$, et al. The importance of long-term acute care hospitals in the regional epidemiology of klebsiella pneumoniae carbapenemaseproducing enterobacteriaceae. Clin Infect Dis. 2013;57:1246-52.

14. Souli M, Galani I, Antoniadou A, Papadomichelakis E, Poulakou G, Panagea T, et al. An outbreak of infection due to betaLactamase Klebsiella pneumoniae Carbapenemase 2-producing K. pneumoniae in a Greek University Hospital: molecular characterization, epidemiology, and outcomes. Clin Infect Dis. 2010;50:364-73.

15. Sexton T, Clarke P, O’Neill E, Dillane T, Humphreys H. Environmental reservoirs of methicillin-resistant Staphylococcus aureus in isolation rooms: Correlation with patient isolates and implications for hospital hygiene. J Hosp Infect. 2006;62:187-94.
16. Ludden C, Cormican M, Vellinga A, Johnson JR, Austin B, Morris D. Colonisation with ESBL-producing and carbapenemase-producing Enterobacteriaceae, vancomycinresistant enterococci, and meticillin-resistant Staphylococcus aureus in a long-term care facility over one year. BMC Infect Dis. 2015; $15: 168$

17. Daszak P, Cunningham AA, Hyatt AD. Emerging infectious diseases of wildlife-- threats to biodiversity and human health. Science. 2000;287:443-9.

18. Davies SC. Annual Report of the Chief Medical Officer. Vol. 2, Infections and the rise of antimicrobial resistance. London, United Kingdom; 2011.

19. Otter JA, Yezli S, French GL. The role played by contaminated surfaces in the transmission of nosocomial pathogens. Infect Control Hosp Epidemiol. 2011;32:687-99.

20. Saba CKS, Amenyona JK, Kpordze SW. Prevalence and pattern of antibiotic resistance of Staphylococcus aureus isolated from door handles and other points of contact in public hospitals in Ghana. Antimicrob Resist Infect Control. 2017;6:44.

21. Clarivet B, Grau D, Jumas-Bilak E, Jean-Pierre H, Pantel A, Parer $\mathrm{S}$, et al. Persisting transmission of carbapenemase-producing Klebsiella pneumoniae due to an environmental reservoir in a university hospital, France, 2012 to 2014. Eur Surveill. 2016;21:30213.

22. Tofteland S, Naseer U, Lislevand JH, Sundsfjord A, Samuelsen $\varnothing$. A long-term low-frequency hospital outbreak of KPC-producing Klebsiella pneumoniae involving intergenus plasmid diffusion and a persisting environmental reservoir. PLoS One. 2013;8:1-8.

23. Walther BA, Ewald PW. Pathogen survival in the external environment and the evolution of virulence. Biol Rev Camb Philos Soc. 2004;79:849-69.

24. Dancer SJ. Controlling hospital-acquired infection: focus on the role of the environment and new technologies for decontamination. Clin Microbiol Rev. 2014;27:665-90.

25. Kramer A, Schwebke I, Kampf G. How long do nosocomial pathogens persist on inanimate surfaces? A systematic review. BMC Infect Dis. 2006;6:130.

26. Weber DJ, Rutala WA, Miller MB, Huslage K, Sickbert-Bennett E. Role of hospital surfaces in the transmission of emerging health care-associated pathogens: Norovirus, Clostridium difficile, and Acinetobacter species. Am J Infect Control. 2010;38: S25-33.

27. Hanski I. Metapopulation ecology. New York, Oxford University Press; 1999.

28. MacArthur RH, Wilson EO. The theory of island biogeography. 14th edn. Princeton: Princeton University Press; 1967.

29. Sax DF, Gaines SD. Equilibrium theory of island biogeography. In: Scheiner SM, Willig MR, (eds). The theory of ecology. Chicago, University of Chicago Press; 2011. p. 219-42.

30. Keeling MJ, Rohani P. Modeling infectious diseases in humans and animals. Princeton, Princeton University Press; 2008.

31. Levins R. Some demographic and genetic consequences of environmental heterogeneity for biological control. Bull Entomol Soc Am. 1969;15:237-40.

32. May RM, Nowak MA. Superinfection, metapopulation dynamics, and the evolution of diversity. $J$ Theor Biol. 1994;170:90-114.

33. Donskey CJ. Does improving surface cleaning and disinfection reduce health care-associated infections? Am J Infect Control. 2013;41:S12-9.

34. Bonten MJ, Austin DJ, Lipsitch M. Understanding the spread of antibiotic resistant pathogens in hospitals: mathematical models as tools for control. Clin Infect Dis. 2001;33:1739-46.

35. van Kleef E, Robotham JV, Jit M, Deeny SR, Edmunds WJ. Modelling the transmission of healthcare associated infections: a systematic review. BMC Infect Dis. 2013;13:294. 
36. Henderson DK, Palmore TN. Critical gaps in knowledge of the epidemiology and pathophysiology of healthcare-associated infections. Infect Control Hosp Epidemiol. 2010;31:S4-6.

37. Weinstein RA, Henderson DK. A double-edged sword and a golden opportunity for healthcare epidemiology. Infect Control Hosp Epidemiol. 2009;30:1-3.

38. Cohen B, Hyman S, Rosenberg L, Larson E. Frequency of patient contact with health care personnel and visitors: Implications for infection prevention. Jt Comm J Qual Patient Saf. 2012;38:560-5.

39. Drlica K. The mutant selection window and antimicrobial resistance. J Antimicrob Chemother. 2003;52:11-7.

40. Feachem RG. Intervention for the control of diarrhoeal diseases among young children: promotion of food hygiene. Bull World Health Organ. 1984;62:467-76.

41. Köser CU, Ellington MJ, Cartwright EJP, Gillespie SH, Brown $\mathrm{NM}$, Farrington $\mathrm{M}$, et al. Routine use of microbial whole genome sequencing in diagnostic and public health microbiology. PLoS Pathog. 2012a;8:1-9.

42. Dennehy JJ, Friedenberg NA, Holt RD, Turner PE. Viral ecology and the maintenance of novel host use. Am Nat. 2006;167:429-39.

43. Kermack WO, McKendrick AG. A contribution to the mathematical theory of epidemics. Proc R Soc A Math Phys Eng Sci. 1927;115:700-21.

44. Lax S, Sangwan N, Smith D, Larsen P, Handley KM, Richardson $\mathrm{M}$, et al. Bacterial colonization and succession in a newly opened hospital. Sci Transl Med. 2017;9:eaah6500.

45. Omenn GS. Evolution in health and medicine Sackler colloquium: evolution and public health. Proc Natl Acad Sci USA. 2010;107 Suppl:1702-9.

46. Pitout JDD, Nordmann P, Poirel L. Carbapenemase-producing Klebsiella pneumoniae, a key pathogen set for global nosocomial dominance. Antimicrob Agents Chemother. 2015;59:5873-84. 